\title{
Evaluation of ultrasonic aluminium degassing by piezoelectric sensor
}

\author{
H. Puga ${ }^{a}$, J. Barbosa ${ }^{\mathrm{a}, *}$, J. Gabriel ${ }^{\mathrm{b}}$, E. Seabra ${ }^{\mathrm{a}}$, S. Ribeiro ${ }^{\mathrm{c}}$, M. Prokic $^{\mathrm{d}}$ \\ ${ }^{a}$ CT2 M - Centre for Mechanical and Materials Technologies, Universidade do Minho, Azurém 4800-058 Guimarães, Portugal \\ b IDMEC - Pólo FEUP, Faculdade de Engenharia da Universidade do Porto, 4200-465 Porto, Portugal \\ ${ }^{\mathrm{c}}$ FEUP, Departamento de Enga Metalúrgica e de Materiais, 4200-465 Porto, Portugal \\ ${ }^{\mathrm{d}}$ MP Interconsulting, 2400 Le Locle, Switzerland
}

\section{A R T I C L E I N F O}

\section{Article history:}

Received 27 July 2010

Received in revised form

23 December 2010

Accepted 8 January 2011

Available online 14 January 2011

\section{Keywords:}

Melting

Degassing

Casting

Aluminium alloys

Piezoelectric devices

\begin{abstract}
A B S T R A C T
The purpose of this work was the development of a reliable technique to evaluate the intensity of acoustic cavitation during degassing of aluminium melts and to use it to select the optimum processing time for an envisaged degassing efficiency.

A high sensitivity piezoelectric disk type device was used as a sensing feedback in water and liquid AlSi9Cu3 alloy. The signal acquisition and processing was carried out on a dedicated LabVIEW ${ }^{\circledR}$ based application which allowed real-time monitoring of the piezoelectric sensor's data and ultrasonic parameters. Standard Fast Fourier Transform was applied to obtain the dominant frequencies, as well as the sub and ultra-harmonics. It was found that the amplitude of the FFT sub-harmonic $(f / 2)$ was the best indicator to evaluate the process degassing efficiency, and it could be used to select the optimal processing time, independently of other variables.

The developed methodology was applied to several AlSi9Cu3 melts, and validated by measuring the final alloy densities and the volume fraction of porosities, revealing that it is an efficient, fast and cost effective technique to evaluate the degassing treatment of aluminium alloys. Experimental curves of AlSi9Cu3 alloy degassing efficiency as a function of $f / 2$ amplitude are presented for different degassing times.
\end{abstract}

(c) 2011 Elsevier B.V. All rights reserved.

\section{Introduction}

The quality of a casting depends directly on the quality of the molten metal used to produce it. Thus, reliable melt treatment techniques, and fast and effective characterization methods to evaluate the quality of molten alloys are crucial aspects for foundrymen.

In what concerns to aluminium alloys, melt treatment includes cleaning, microstructure refinement/modification and degassing. Techniques to evaluate treatment efficiency and to characterize melt quality often require laboratory analysis of multiple samples taken from the molten alloy. This procedure is detrimental for process efficiency, since samples production and characterization are time consuming, and melt conditions can change over the time. On-line monitoring of the melt quality can be a faster procedure, but applications known so far are limited to lab scale chemical composition monitoring using laser induced breakdown spectroscopy (Kai et al., 2002) or chemical sensors based on galvanic cells (Vangrunderbeek et al., 1999; Fray, 1996), inclusion detection by electromagnetic (Makarov et al., 1999; Roderick et al., 2001) or ultrasound techniques (Ono et al., 2002, 2004) and mea-

\footnotetext{
* Corresponding author. Tel.: +351 253510220/59; fax: +351 253516007

E-mail address: kim@dem.uminho.pt (J. Barbosa).
}

surement of hydrogen concentration in molten aluminium alloys either by using highly unstable galvanic cells (Schwandt et al., 2003; Zheng and Zhen, 1993) or very slow carrier gas techniques like AlScan or Telegas (Fergus, 2005).

One of the main problems concerning to aluminium foundry practice is porosity, which is highly detrimental to the mechanical and fatigue performance of a casting. Although it can be due to factors associated to volume shrinkage compensation during solidification, porosity in $\mathrm{Al}$ alloys is generally caused by precipitation of hydrogen during cooling, since a large drop in solubility occurs at solidification point (Gruzleski and Closset, 1990).

During the last years, ultrasound has found application in many metallurgical related areas, like machining (Singh and Khamba, 2006), welding (Matsuoka and Imai, 2009), 3D direct manufacturing (Kong et al., 2004) and foundry (Puga et al., 2009a,b). Among those applications, ultrasonic (US) degassing, an extremely efficient and environmentally friend degassing technique, has been under development during the last years by several researchers (Meidani and Hasan, 2004; Xu et al., 2008; Puga et al., 2009a,b) and it is now ready for industrial widespread application. It is based on the supply of acoustic energy to the molten metal in order to induce cavitation. According to Eskin (1998), when a liquid metal is submitted to high intensity ultrasonic vibrations, the alternating pressure above the cavitation threshold creates numer- 
ous cavities in the liquid metal associated to noise development, which intensifies mass transfer processes and accelerates the diffusion of hydrogen from the melt to the developed bubbles. As acoustic cavitation progresses with time, adjacent bubbles touch and coalesce, growing to a size sufficient to allow them to rise up through the liquid, against gravity, until reaching surface, as suggested by Meidani and Hasan (2004) and Eskin (1998). Nevertheless, many of those bubbles grow and collapse before reaching the liquid surface, creating new nucleation sites and adding to the acoustic signal not only a main frequency, but also several harmonics, sub-harmonics and incoherent noise, as demonstrated by Eskin (1998). Although the high efficiency and hydrogen removal rate of US degassing, measurement of hydrogen concentration or alloy density evaluation is time consuming, and can take several minutes using traditional measurement techniques. Moreover, the accurate control of US parameters to achieve good results is difficult, since the process efficiency depends on many factors, such as melt temperature, US frequency, electric energy converted into acoustic energy, processing time and acoustic wave attenuation, and technicians' experience. For such reasons, the development of a reliable method/technique to evaluate degassing results is of high interest for the aluminium foundry industry.

Although the large amount of processing variables, high degassing rate is only possible if a well-developed cavitation regime is developed inside the aluminium melt, as suggested by Puga et al. (2009), Eskin (1998) and Abramov (1998). This is therefore the key issue of the process that can make ultrasonic degassing an easy to operate, simple and efficient technique.

In this work, a cavitation monitoring technique based on the vibration measurement was used to evaluate the required processing time for an envisaged degassing efficiency or final alloy density. For that purpose, a piezoelectric device was used to measure the vibration generated by acoustic cavitation, and tested in water and liquid AlSi9Cu3 alloy. Piezoelectric materials have been used for a long time in numerous applications, whether as sensors, or as actuators. As a sensor, a piezo device has several advantages over other concurrent solutions, it has a very fast response time (in the order of microseconds) and a large bandwidth, it is relatively cheap and it does not need power to work. In fact, piezoelectric materials have the ability to develop an electric charge proportional to a direct applied mechanical stress, and therefore are likely to be used as vibration sensors.

\section{Degassing system}

\subsection{Acoustic sensor}

In this application it was used the piezo KPSG-100 (Kingstate) as an acoustic sensor. This piezo disk is made of a ceramic layer of about $23.5 \mathrm{~mm}$ diameter and $1 \mathrm{~mm}$ thickness, deposited over a thin disk made of brass. Piezo ceramics of this type are advantageous over quartz or polymers since they present high sensitivity producing a larger amplitude signal for the same vibration. A piezo disk is a low cost, yet high performance solution to measure vibrations in the $\mathrm{kHz}$ range, as it is the case. The piezo disk was coupled to the top of a molybdenum rod with $2 \mathrm{~mm}$ diameter and $1000 \mathrm{~mm}$ length, which was inserted in the liquid were acoustic vibrations were going to be measured.

This piezo is a disk poled in directions 31 (Fig. 1(a)) and 33 (Fig. 1(b)), meaning that it can generate electrical charges from these two mechanical stress directions. (1):

For the case (a), the generated voltage can be estimated by Eq.

$V_{31}=\frac{g_{31} \times F_{1}}{2 \times \pi \times r}$

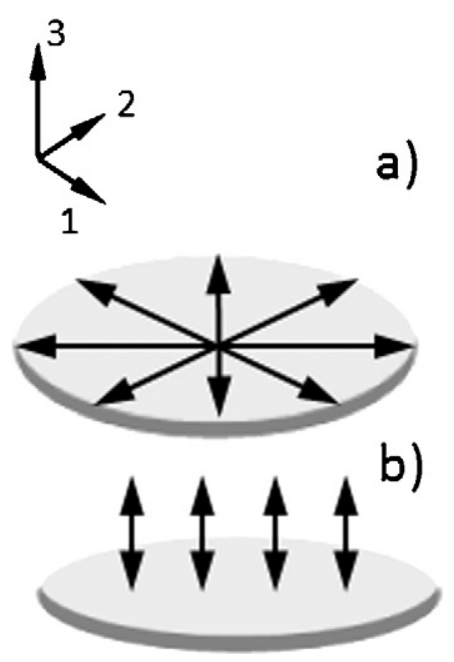

Fig. 1. Piezo disk axis and stress directions.

where $g_{31}$ is the electric field generated by the applied stress in the direction $1, F_{1}$ is the applied force (in the direction 1 ) and $r$ is the radius.

For the case (b), the equation comes (2):

$V_{33}=\frac{g_{33} \times F_{3} \times t}{\pi \times r^{2}}$

where $g_{33}$ is the electric field generated by the applied stress in the direction $3, F_{3}$ is the applied force (in the direction 3 ) and $t$ is the thickness.

A piezoelectric material presents complex impedance and its behavior changes with the frequency. The material is usually modeled through a Butterworth Van-Dyke configuration, which includes three parameters: capacitance, inductance and resistance. These values were measured using a LCR meter (Escort, ELC-131D) at a frequency of $1 \mathrm{kHz}$ having obtained, respectively $C=51.98 \mathrm{nF}$, $L=483 \mathrm{mH}$, and $R=78.47 \mathrm{k} \Omega$.

To acquire the output signal of a piezo sensor it is common to use a so-called charge amplifier. This electronic circuit includes a high input impedance (low bias current) amplifier in parallel with a small capacitance. However, since the data acquisition board used in this work presented already high impedance (10G $\Omega$ ), and the signal to read had high frequency, it was directly connected to the board.

\subsection{Ultrasonic generator}

Most of the traditional ultrasonic applications are based on fixed-frequency, well-tuned ultrasonic sources, where a large number of designs and matching parameters must be respected (Xu et al., 2008; Eskin, 1998). Extensive field tests have demonstrated (Eskin, 1998; Prokic, 2004) that in order to achieve high efficiency, the ultrasonic systems must be well tuned to the load. Since most ultrasound units work inherently in non-stationary conditions, in theory they have to continuously adapt themselves to the load to maximize the efficiency, which is difficult to achieve with the fixed-frequency units.

On this work, a novel MMM (Multi-frequency Multimode Modulated technology) ultrasonic technique developed by MP Interconsulting (Prokic, 2001) was used. MMM technology is characterized by synchronously exciting many vibration modes through the coupled harmonics and sub-harmonics in solids and liquid containers. This technology produces high intensity multimode vibration that are uniform and repeatable, which avoid the creation of stationary and standing waves, so that the 


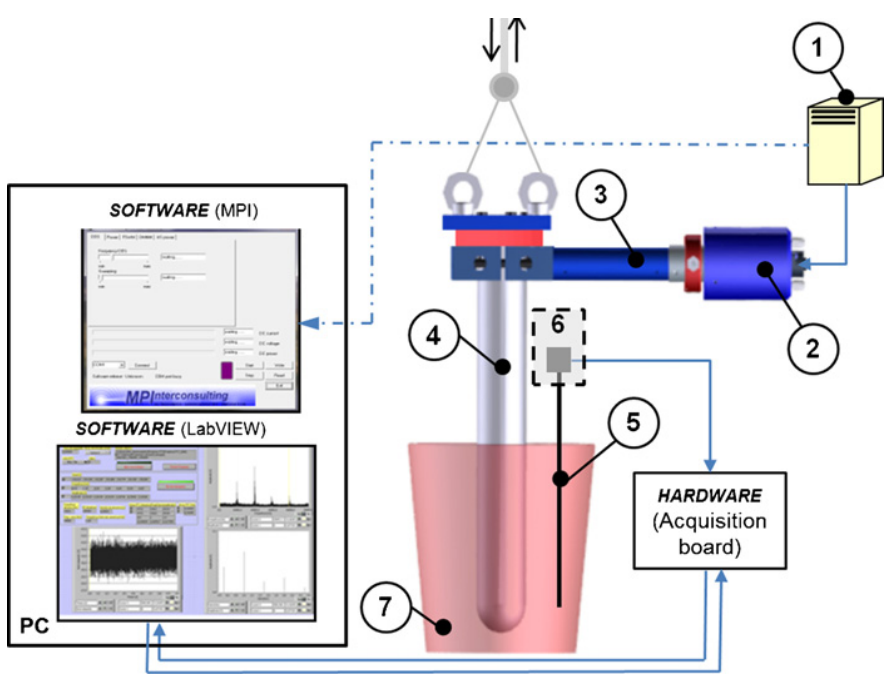

Fig. 2. Schematic of experimental apparatus for measuring the cavitation level in US degassing: 1, ultrasonic power supply unit; 2 , US converter; 3 , waveguide; 4, acoustic radiator; 5 , Mo rod; 6 , piezo disk; 7 , liquid media.

whole vibrating system is fully agitated, improving the degassing process.

\subsection{Experimental set-up}

The experimental set-up (Fig. 2) used to measure the signal of acoustic cavitation during the ultrasonic degassing of aluminium alloys consisted of a high power ultrasonic converter $(1200 \mathrm{~W})$, a $30 \mathrm{~mm}$ diameter and $150 \mathrm{~mm}$ long acoustic waveguide made of Ti6Al4V, a $60 \mathrm{~mm}$ diameter Ti-based acoustic radiator, a sweepingfrequency, adaptively modulated waveform generated by an MMM ultrasonic power supply unit, and the acoustic sensor presented in Section 2.1 .

The ultrasonic power supply unit is fully controlled by dedicated Windows compatible software developed by MPI. Optimal ultrasonic parameters: sweeping and fswm (frequency shift with modulation) for the selected resonance frequency and electric power are adjusted in order to produce the highest acoustic amplitude and the wide frequency spectrum in the melt, which is monitored with a specifically implemented feedback loop.

The waveform generated by the ultrasonic power supply unit (1) is converted in repetitive multi-frequency mechanical vibrations by the ultrasonic converter (2), which are driven to the acoustic radiator (4) through the waveguide (3). The acoustic load (radiator and liquid) starts producing its own vibration, oscillating in one or more of its natural resonance frequencies.

The vibrations and shock waves developed by cavitation in the liquid media are transmitted to the molybdenum rod (5) inserted in the liquid (7), and then converted into an electrical signal proportional to the mechanical stress by the coupled piezo disk (6).

The piezoelectric signal was acquired by an analogue channel of a PCI-6251 board from National Instruments at a rate of 200,000 samples/s. For each power level tested, the spectrum was calculated 10 times, and the average values evaluated to minimize random errors.

A suitable LabVIEW ${ }^{\circledR}$ based application was developed for data acquisition, analysis and signal processing. Data processing consists in the spectral analysis of the piezoelectric signal, using the LabVIEW $^{\circledR}$ routines for Fast Fourier Transform (FFT).

The program does the data processing of files logged in disk, composed by the voltage values read from the piezo sensor. The data is processed by using the routine FFT.VI, from the LabVIEW ${ }^{\circledR}$ library. This routine computes the Fast Fourier Transform (FFT) of the input sequence, giving the Real FFT vector as output. From the sampling rate, two vectors are computed: one that contains the time for each sample and another that contains the frequency. In the interface, the user can define the update time, as well as the sampling rate and number of samples to acquire. As output, the interface presents the processed data and three graphs: time/amplitude, frequency/amplitude, and dominant frequencies/amplitude.

Although FFT analysis is a classical method of data processing, its implementation in a LabVIEW ${ }^{\circledR}$ routine made it possible the automatic calculation of the harmonics, sub and ultra-harmonics amplitude, which are visualized almost in real time and used to evaluate the cavitation intensity in molten metal, thus anticipating the required degassing time. This is the main novelty of the use of FFT in this research.

\subsection{Experimental procedure}

To evaluate the cavitation signal, tests were carried out in water and in molten AlSi9Cu3 alloy. Water is considered a suitable media to simulate US degassing mechanism in liquid aluminium alloys (Eskin, 1998), and it was used for the first series of tests, in order to visually identify the development of cavitation bubbles. A glass recipient with $180 \mathrm{~mm}$ diameter and $200 \mathrm{~mm}$ height, filled with $3 \mathrm{l}$ of water at $18 \pm 1^{\circ} \mathrm{C}$ was used to simulate the melting crucible. Energy power up to $700 \mathrm{~W}$ with $50 \mathrm{~W}$ increments at a frequency of $19,800 \pm 100 \mathrm{~Hz}$ was used to promote cavitation, and the acoustic sensor signal was recorded.

After characterizing the cavitation signal in water, melting stocks of AlSi9Cu3 alloy weighing $4 \mathrm{~kg}$ were melted in a resistance furnace equipped with a $170 \mathrm{~mm}$ diameter and $180 \mathrm{~mm}$ height $\mathrm{SiC}$ crucible. Melt temperatures of 700 and $780^{\circ} \mathrm{C}$ within an accuracy of $\pm 10^{\circ} \mathrm{C}$ were used to perform cavitation tests, using the same ultrasonic parameters that were used for water. Samples for characterization were taken immediately before starting the degassing operation and after 1, 3 and 5 min ultrasonic processing for each processing parameters combination. The Reduced Pressure Test (RPT) and the apparent density measurement method were used to evaluate samples density. The volume fraction of porosities was evaluated by digital imaging techniques, using Image-Pro Plus software.

The initial densities of molten alloys (corresponding to those samples taken immediately before starting the degassing operation) were in the range between 2.4 and $2.45 \mathrm{~kg} \mathrm{dm}^{-3}$. The degassing efficiency $\eta$ was calculated according to the following expression:

$\eta=\frac{D_{f}-D_{i}}{D-D_{i}} \times 100$

where $D$ is the theoretical alloy density $\left(2.74 \mathrm{~kg} \mathrm{dm}^{-3}\right)$ and $D_{i}$ and $D_{f}$ are the initial and final alloy densities, respectively.

\section{Results and discussion}

Fig. 3 shows an example of a typical sound pressure waveform as received by the piezoelectric.

Fig. 4(a)-(c) shows the FFT spectral levels of harmonics, sub and ultra-harmonics of acquired sound pressure waveforms, for 250 360 and $600 \mathrm{~W}$ electric power, respectively. According to Abramov (1998) the intensity of the FFT sub-harmonic $(f / 2)$ is the most reliable parameter to evaluate the development of cavitation in liquid metals. Moreover, when the intensity of the FFT sub-harmonic increases and the ultra-harmonics signal stabilizes, a regime of well developed cavitation is achieved (Abramov, 1998). According to Fig. 4(a), it is clear that for $250 \mathrm{~W}, 3 / 2 f$ and $2 f$ ultra-harmonics (corresponding to approximately 30 and $40 \mathrm{kHz}$, respectively) are still not stabilized, suggesting that the cavitation threshold has not 


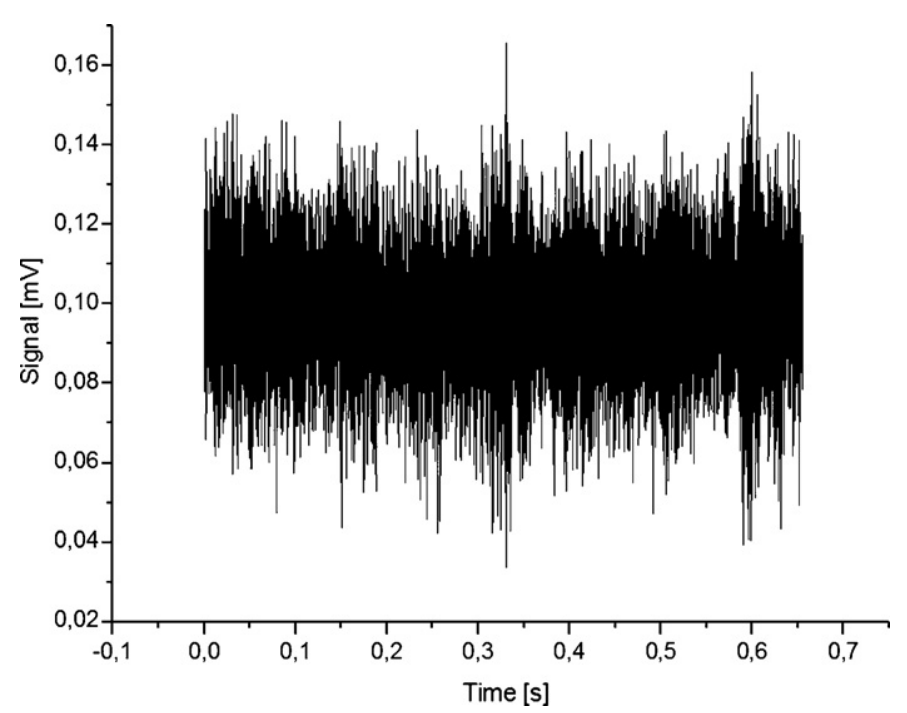

Fig. 3. An example of a typical sound pressure waveform.

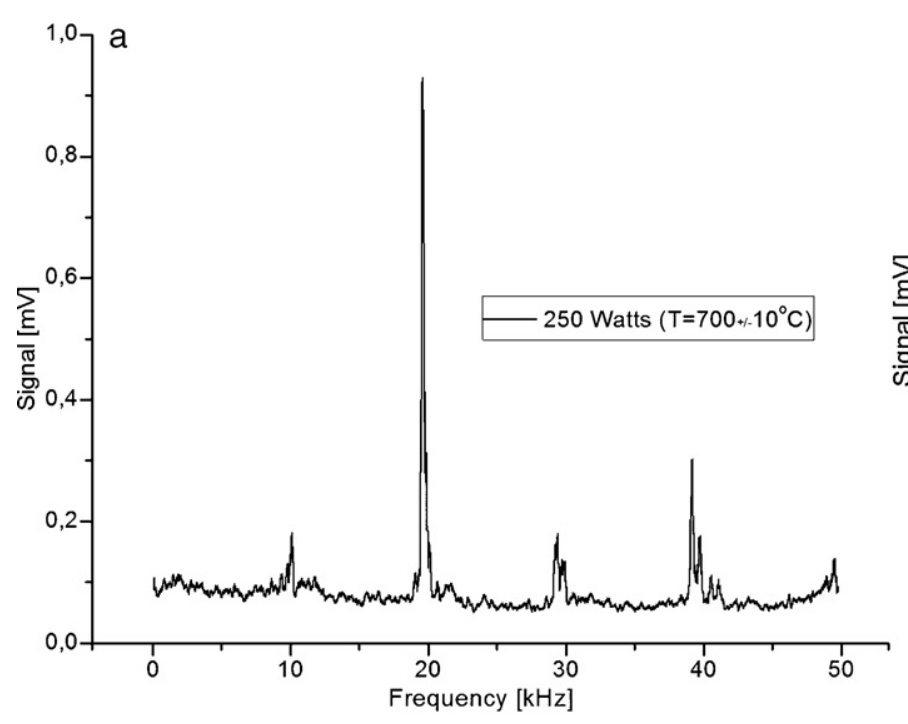

been reached. However, for 360 and $600 \mathrm{~W}$ (Fig. 4(b and c)) not only the ultra-harmonics are stabilized, but also the $f / 2$ sub-harmonics (corresponding to approximately $10 \mathrm{kHz}$ ) are fully developed and increase with electric power, suggesting that a developed cavitation regime has been reached.

In Fig. 5, the sub-harmonic intensity is plotted against the electric power, and 3 regions can be clearly distinguished: Zone 1 , corresponding to insipient cavitation; Zone 2 corresponding to the cavitation threshold; Zone 3 corresponding to a well developed cavitation regime. The transition from Zone 1 to Zone 3 was characterized by a sharp increase in the acoustic signal, indicating the cavitation threshold, which agrees with suggestions of Eskin (1998) and Abramov (1998).

Fig. 5(a) corresponds to cavitation experiment in water at room temperature, and shows the variation of the sub-harmonic $(f / 2)$ amplitude as a function of electric power. Each point is the average of 10 readings for each value of electric power. Between 270 and $300 \mathrm{~W}$ electric power, a sudden increase in the sub-harmonic amplitude was detected, and a change in the curve slope was clearly identified. This means that the cavitation threshold was reached and for higher values a well-developed cavitation regime was established, which was visually confirmed by a sudden increase in the amount of cavitation bubbles and the development of acoustic

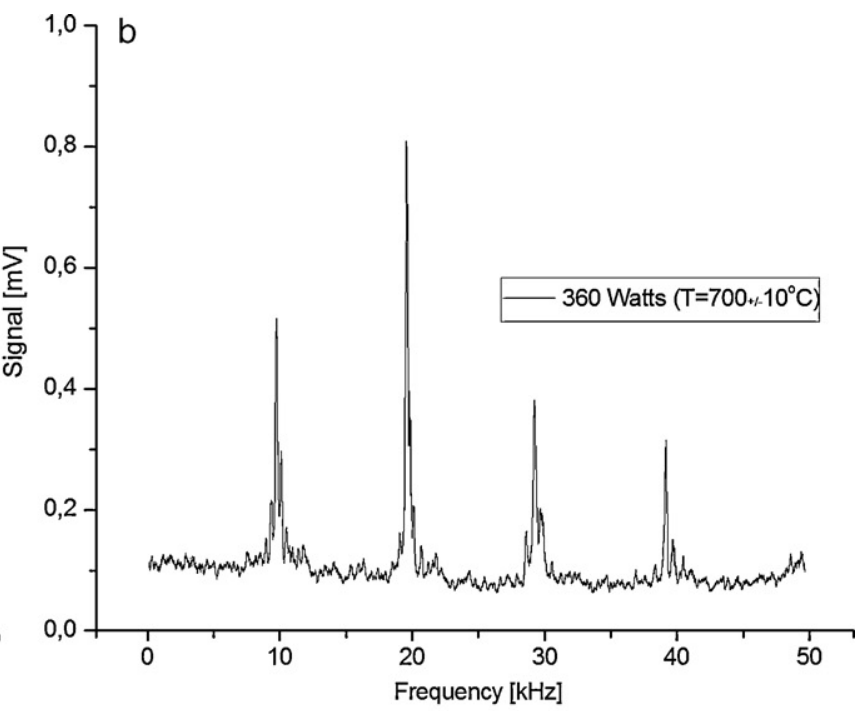

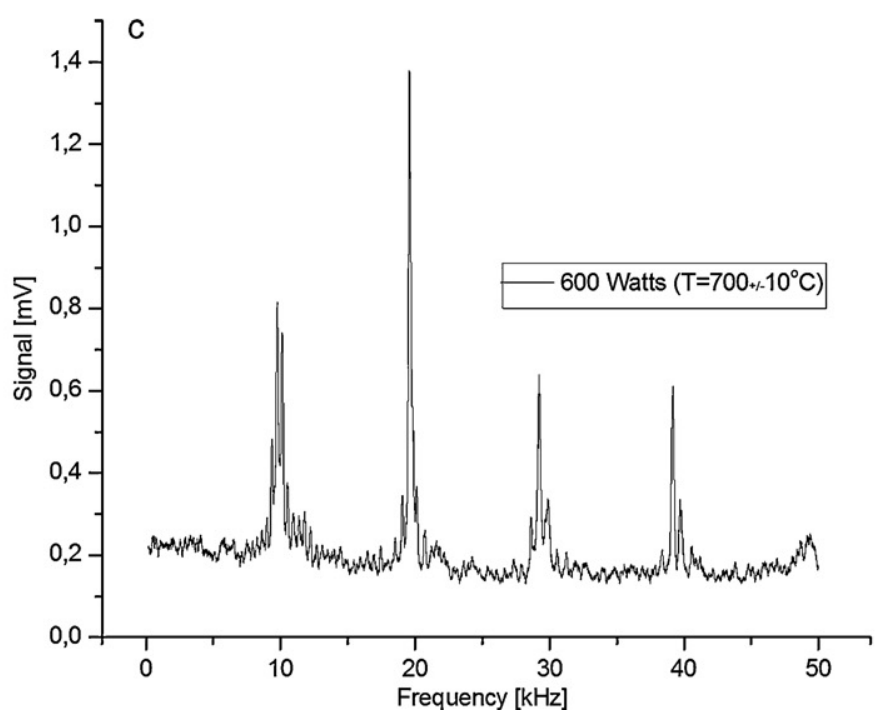

Fig. 4. FFT spectral levels of harmonics, sub and ultra-harmonics of sound pressure waveform, for (a) $250 \mathrm{~W}$, (b) $360 \mathrm{~W}$ and (c) $600 \mathrm{~W}$ electric power. 

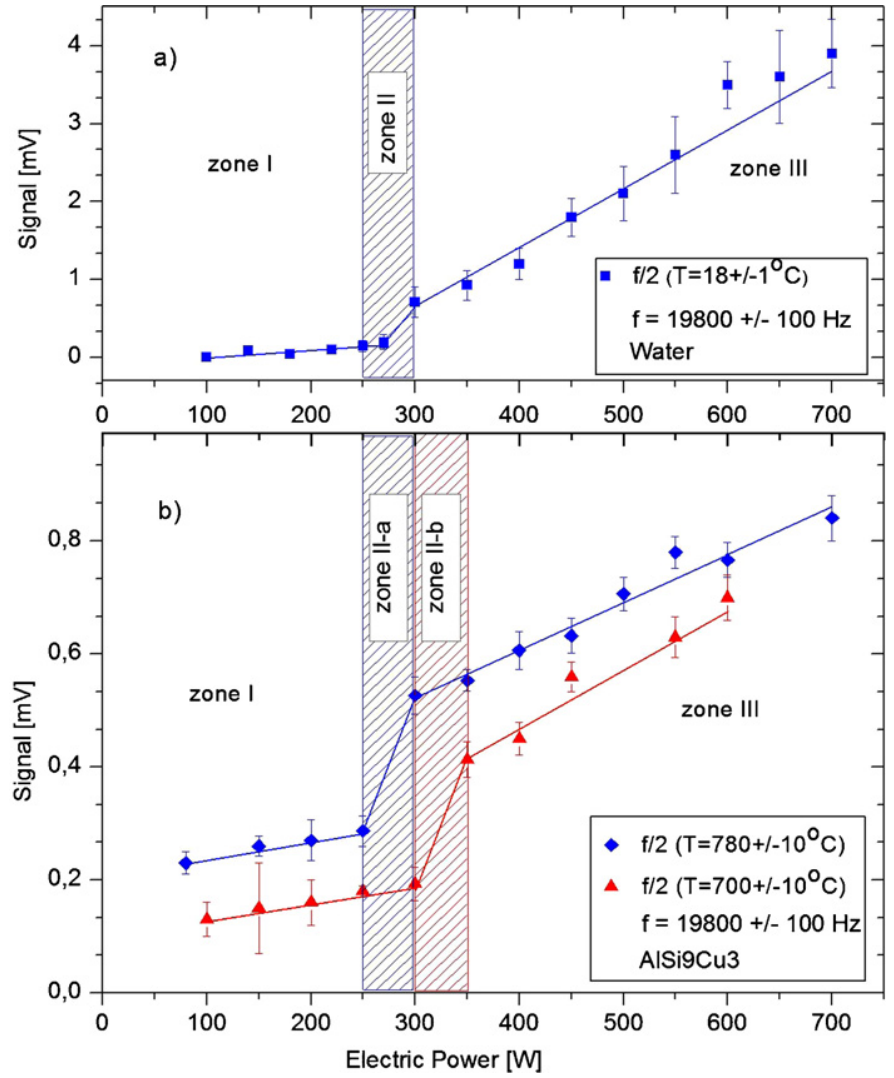

Fig. 5. Amplitude of sub-harmonics as a function of electric power: (a) in water and (b) in molten AlSi9Cu3.

streams, confirming previous suggestions of Brabec and Mornstein (2007) concerning to tests performed with water. This behavior also confirmed the findings of Abramov (1998) concerning to the relation between the sub-harmonic $(f / 2)$ amplitude and cavitation intensity.

Fig. 5(b) corresponds to cavitation experiments in AlSi9Cu3, and shows the variation of the sub-harmonic $(f / 2)$ amplitude as a function of electric power, for 700 and $780^{\circ} \mathrm{C}$ melt temperature. The curves presented the same development of that observed for experiments in water, but the signal amplitudes were lower due to acoustic wave attenuation. Temperature increase shifted Zone 2 to the sense of lower electric power and increased the signal amplitude, due to the decrease in both the alloy viscosity and acoustic wave attenuation. Minimum sub-harmonic amplitudes corresponding to a well-developed cavitation regime were $0.4 \pm 0.03$ and $0.53 \pm 0.03 \mathrm{mV}$ for 700 and $780^{\circ} \mathrm{C}$, respectively. From Fig. 5(b) it is also clear that similar $f / 2$ amplitudes could be achieved for different electric power values if different melt temperatures were used, revealing that the cavitation intensity, is a function of different processing parameters.

In Fig. 6 the alloy density was plotted as a function of processing time, for values of electric power that fall within the zone of welldeveloped cavitation $(600 \mathrm{~W})$, at the start of that zone $(360 \mathrm{~W})$ and at start of the transition zone ( $250 \mathrm{~W})$, for $19.8 \mathrm{kHz}$ frequency and 700 and $780^{\circ} \mathrm{C}$ melt temperature.

It was clear that for those samples obtained at $250 \mathrm{~W}$, the degassing efficiency was very low, as density did not reach a steady state plateau, even after 5 min processing and remained very far from the theoretical AlSi9Cu3 alloy density of $2.74 \mathrm{~kg} \mathrm{dm}^{-3}$. This was due to the low density of cavitation bubbles, which slew down the hydrogen removal rate. Electric powers of 360 and $600 \mathrm{~W}$ promoted high degassing level, due to the formation of higher num-

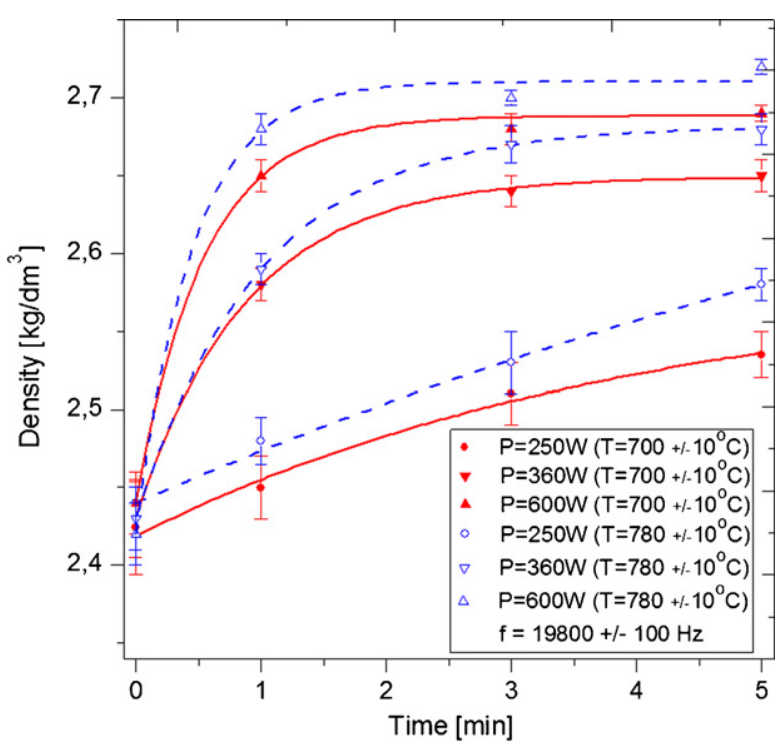

Fig. 6. AlSi9Cu3 alloy density as a function of processing time, for different values of electric power and melt temperature.

ber of cavitation bubbles and the partial displacement of drops of liquid in the treated volume. As a consequence, strong acoustic streams developed and improved the coagulation of separate bubbles of hydrogen and its floating to the surface of the pool. Consequently a raise of the degassing and density increase rates occurred, and the alloys density reached the maximum values of 2.71 and $2.68 \mathrm{~kg} \mathrm{dm}^{-3}$ after 1.5 and 4 min degassing for 600 and $360 \mathrm{~W}$ electric power, respectively.

Experimental results also revealed that the maximum alloy density and degassing rate increased with the melt temperature, for the same electric power. The alloy density increase was a consequence of a reduction on the volume fraction of porosity, which decreased with the increase of both the electric power and melt temperature (Figs. 7 and 8), thus with the intensity of cavitation and the cavitation noise (as suggested by Fig. 5(b)). Fig. 8 shows the macrostructure of RPT samples cross-sections corresponding to points $1-4\left(700^{\circ} \mathrm{C}\right.$ curve), identified in Fig. 7. It is perceptible that only the sample processed under a well-developed cavitation regime (see Fig. 5(b)) does not reveal the presence of porosities. Moreover, the volume fraction of porosities increases as the ampli-

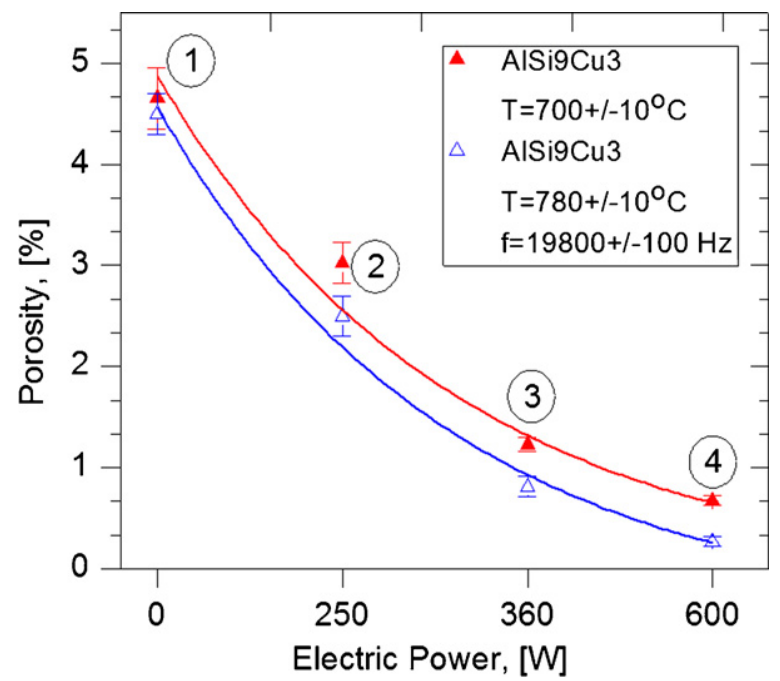

Fig. 7. Volume fraction of porosity as a function of elecric power, for 700 and $780^{\circ} \mathrm{C}$ melt temperature. 

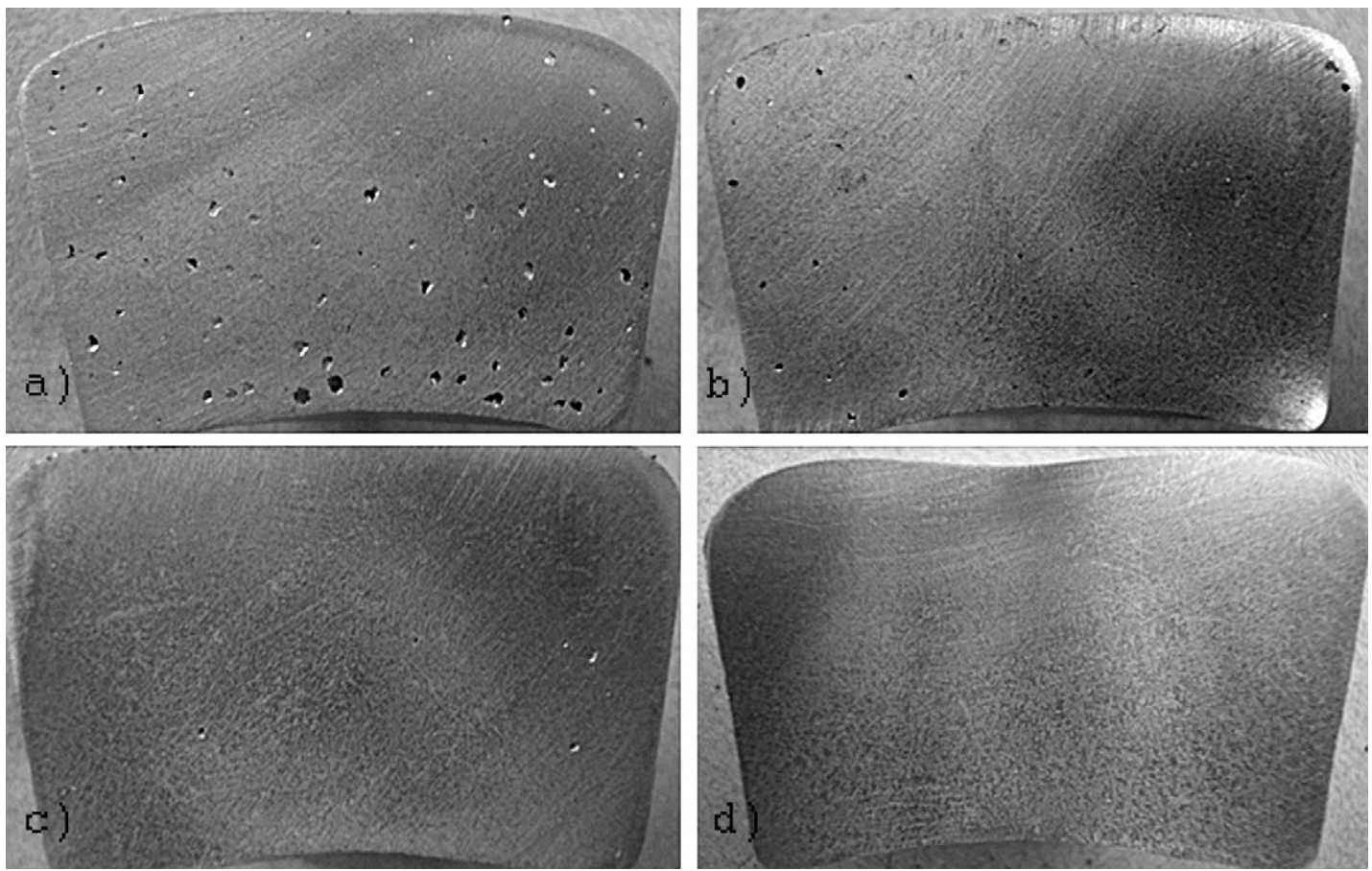

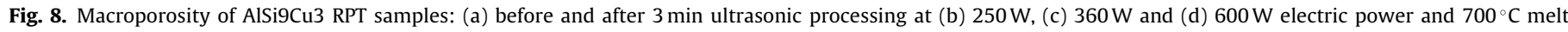
temperature.

tude of the sub-harmonic $(f / 2)$ decreases and the cavitation regime gets close to Zones 2 and 1 . These results are a valuable indication that the degassing efficiency and the castings porosity level can be related with the signal of the acoustic sensor, by using the amplitude of the spectra FFT $f / 2$ sub-harmonic as a feedback variable.

Density values of RPT samples were used to evaluate the degassing efficiency for each sub-harmonic $(f / 2)$ amplitude, and different holding times. This way, degassing efficiency was directly related with that parameter, which was monitored in real time, and results are graphically presented in Fig. 9. Shadowed area represents the usual envisaged degassing efficiency range for cast aluminium alloys.

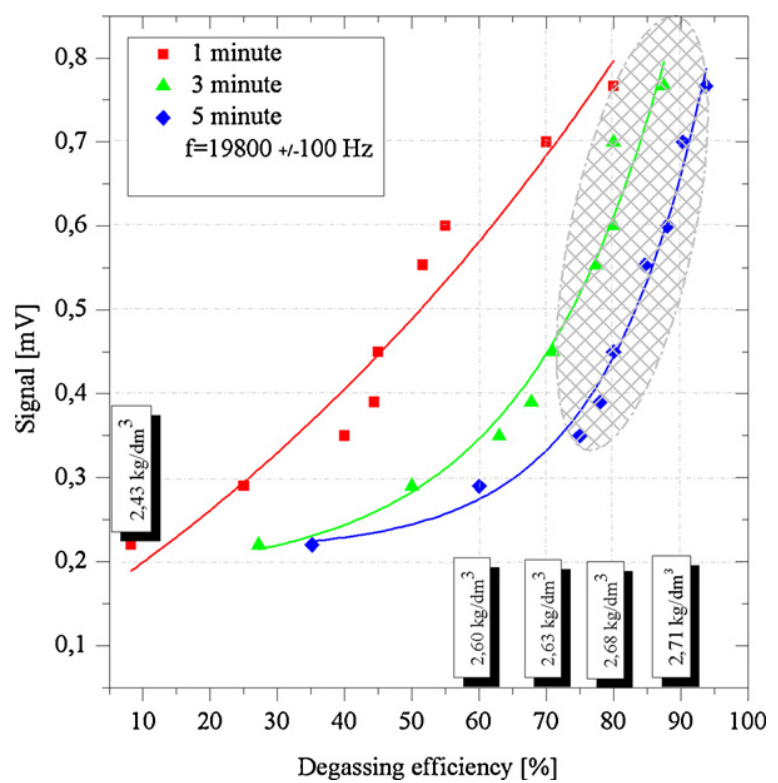

Fig. 9. Degassing efficiency as a function of spectra FFT sub-harmonic amplitude for the AlSi9Cu3 alloy, for different degassing times.
An exponential regression was carried out using OriginLab ${ }^{\circledR}$ software to obtain a best-fit curve relating the $f / 2$ amplitude and degassing efficiency values, for each processing time. The obtained general function, with correlation coeficients between $93 \%$ and $98 \%$, is the following:

$y=y_{0}+A \times e^{-x / t}$

Which can be transformed into:

Signal $=$ Signal $_{0}+K_{1} \times e^{-E f f i c i e n c y /} K_{2}$

where Signal is the $f / 2$ amplitude, Signal $_{0}$ is the initial $f / 2$ amplitude (in this case it corresponds to approximately $0.22 \mathrm{mV}$, see Fig. 9), $K_{1}$ and $K_{2}$ are constants and Efficiency is the degassing efficiency.

In Table 1 the values of $K_{1}, K_{2}$ and the correlation coefficient for each curve represented in Fig. 9 are presented.

In order to validate this model, RPT samples taken after 1, 3 and 5 min degassing, when $f / 2$ amplitude was $0.45 \mathrm{mV}$, were sectioned to evaluate porosity, and their macrostructures are presented in Fig. 10(a)-(c).

It is clear that 1 min degassing (Fig. 10(a)) was not enough to eliminate most porosities, since large pores are still visible all over the sample. The sample taken after 3 min degassing (Fig. 10(b)) reveals some small cavities, representing a volume fraction much lower than the sample taken after 1 min degassing. Sample taken after 5 min degassing (Fig. 10(c)) does not reveal any porosity, suggesting that the degassing efficiency was very close to $100 \%$. If we locate these samples in Fig. 9, we can see that only that sample taken after 5 min degassing (Fig. 10(c)) falls within the shadowed area

Table 1

Constants and correlation coefficient $\left(R^{2}\right)$ of Eq. (5)

\begin{tabular}{lccc}
\hline Parameters & \multicolumn{2}{l}{ Time (min) } \\
\cline { 2 - 4 } & 1 & 3 & 5 \\
\hline$R^{2}(\%)$ & 93 & 95 & 98 \\
$K_{1}$ & 0.54 & 0.01 & 0.001 \\
$K_{2}$ & -100 & -21 & -15 \\
\hline
\end{tabular}


a

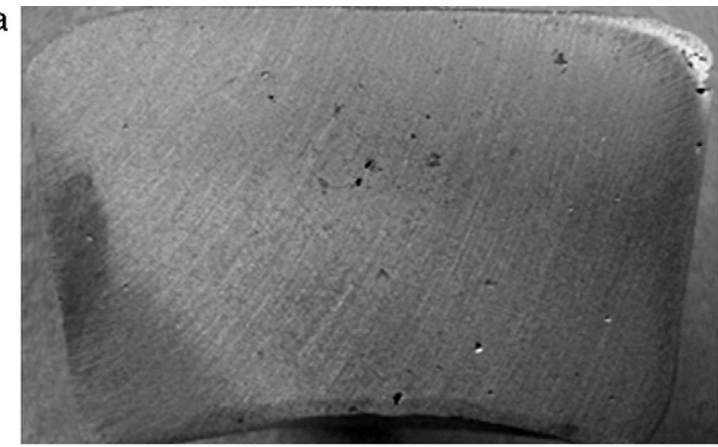

b

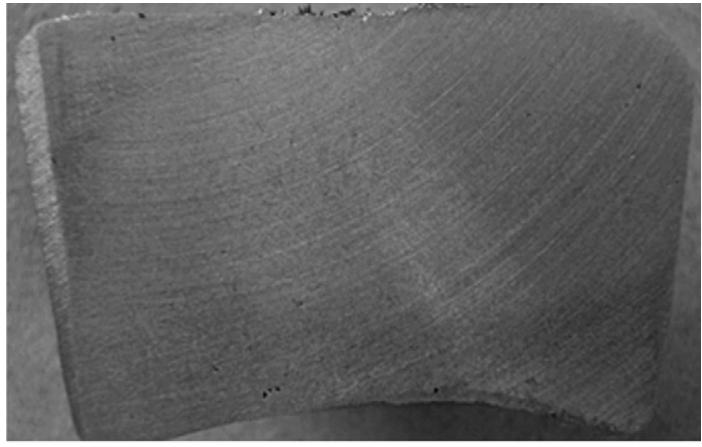

C

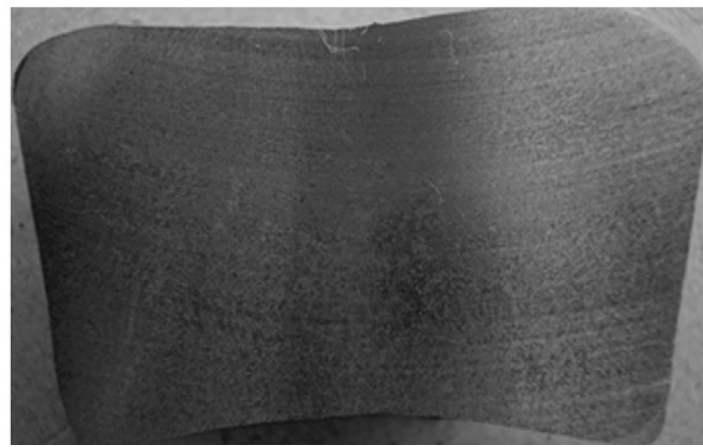

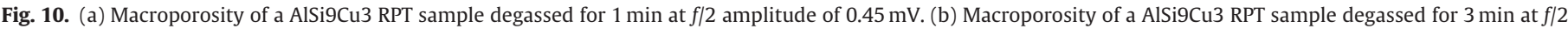
amplitude of $0.45 \mathrm{mV}$. (c) Macroporosity of a AlSi9Cu3 RPT sample degassed for 5 min at $\mathrm{f} / 2$ amplitude of $0.45 \mathrm{mV}$.

that represents an acceptable degassing efficiency. Moreover, the sample taken after 3 min degassing is located at the border of the shadowed area, meaning that an acceptable efficiency was almost achieved, but some porosities could still appear, which is in agreement with the sample porosity level shown in Fig. 10(b). Finally, the sample taken after 1 min degassing is deeply located at an unefficient degassing location, corresponding to approximately $40 \%$ degassing efficiency, confirming the result observed in Fig. 10(c).

Macroporosity evaluation is a good indicator of the level of degassing of a RPT sample. However it gives no information concerning to microporosity. In Fig. 11, the microstructure of two RPT samples, corresponding to 1 and 3 min degassing with subharmonic $(f / 2)$ amplitude of $0.7 \mathrm{mV}$ (corresponding to locations of expected acceptable and not acceptable degassing efficiencies, respectively - see Fig. 9), is presented. A high volume fraction of porosities is present in that sample degassed for $1 \mathrm{~min}$, but in the sample degassed for $3 \mathrm{~min}$ microporosity is very low.
These results, namely the level of porosity obtained in those tests performed to validate the model, suggest that the developed method is reliable and can be used to estimate the degassing level of a molten $\mathrm{Al}$ alloy, exclusively based in the cavitation intensity and degassing time. Moreover, the degassing time can be established based exclusively in the cavitation intensity, namelly the $f / 2$ amplitude of the FFT spectra of the pressure waveform. Curves presented in Fig. 9 are characteristic of a specific processing unit, and a specific aluminium alloy. They can be used to preview the required processing time to reach an envisaged degassing efficiency, for a certain level of cavitation, without taking into consideration any other processing variable. This kind of chart can be produced for every $\mathrm{Al}$ alloy processed by a foundry plant, and be used as a tool to perform ultrasonic degassing.

By using the developed technique it is possible to control the efficiency of the degassing process without carrying out RPT samples characterization tests, which is currently the mostly used
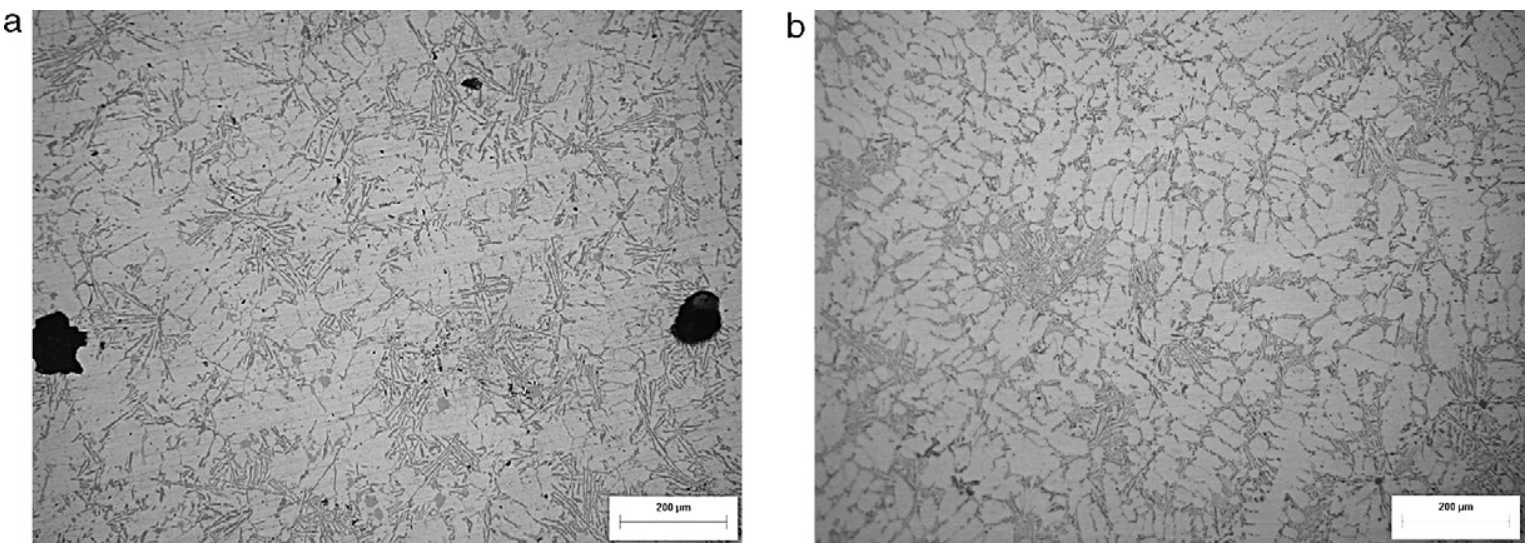

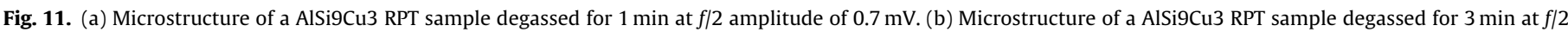
amplitude of $0.7 \mathrm{mV}$. 
procedure to evaluate the amount of dissolved gas in Al alloys. RPT samples evaluation is time consuming, since it often requires the characterization of consecutive samples to adjust the envisaged alloy density. Other techniques, like hydrogen concentration measurement, can take 10-20 min (Fergus, 2005) or being highly unreliable due to the poor stability of solid electrolyte based sensors (Schwandt et al., 2003; Zheng and Zhen, 1993). Moreover, the new technique avoids the use of tools to collect molten samples, which is often a potential cause of hydrogen re-introduction in the liquid metal in industrial practice. These aspects are the main novelties of the developed methodology when compared to current industrial practices.

\section{Conclusions}

A reliable technique to control ultrasonic degassing of Al melts was developed. It was found that the treatment time to reach a certain degassing efficiency can be predicted based exclusively on the amplitude of the spectra FFT sub-harmonic, obtained from the sound pressure waveform spectrum acquired in real-time by the piezoelectric disk. In addition, it can also be referred that:

- The efficiency of ultrasonic degassing can be evaluated by controlling the degassing time for a known amplitude of the spectra FFT sub-harmonic $(f / 2)$.

- The amplitude of the spectra FFT sub-harmonic $(f / 2)$ depends on several factors, namely the electric power and the melt temperature.

- The degassing efficiency is an exponential function of the amplitude of the spectra FFT sub-harmonic $(f / 2)$.

- During the ultrasonic degassing of Al alloys, 3 zones of cavitation can be clearly identified. The transition from insipient cavitation to a fully developed cavitation regime is characterized by a sharp increase in the amplitude of the FFT sub-harmonic $(f / 2)$.

In addition, for the specific processing conditions used in this work, the following conclusions can also be taken concerning the degassing efficiency:

- The start value of zone 3 corresponding to a well-developed cavitation regime is $0.4 \mathrm{mV}$ for $360 \mathrm{~W}$ at $700{ }^{\circ} \mathrm{C}$ and $0.53 \mathrm{mV}$ for $300 \mathrm{~W}$ at $780^{\circ} \mathrm{C}$. For higher power levels, the sub-harmonic $(f / 2)$ amplitude increases, reaching 0.68 and $0.78 \mathrm{mV}$, respectively for 700 and $780^{\circ} \mathrm{C}$, at $600 \mathrm{~W}$.

- For $600 \mathrm{~W}$ and $780^{\circ} \mathrm{C}$, the AlSi9Cu3 alloy density reaches a maximum value of $2.71 \mathrm{~kg} \mathrm{dm}^{-3}$ after $1.5 \mathrm{~min}$ degassing. For $700{ }^{\circ} \mathrm{C}$ that value decreases to $2.68 \mathrm{~kg} \mathrm{dm}^{-3}$ after 2 min degassing. The volume fraction of porosity associated to both samples is $0.24 \%$ and $0.68 \%$, respectively.

- For sub-harmonic $(f / 2)$ amplitudes less than $0.33 \mathrm{mV}$, it is not possible to obtain porosity free samples. The minimum degassing time is 5, 3 and $1 \mathrm{~min}$ which corresponds to $0.40,0.55$ and $0.77 \mathrm{mV}$ sub-harmonic ( $f / 2$ ) amplitude, respectively.

The developed method is original and innovative, and can be used to estimate the degassing level of an Al alloy, exclusively based in the cavitation intensity and degassing time.

\section{Acknowledgments}

This research was supported by FCT - Portuguese Foundation for Science and Technology and was developed on the aim of the research project PTDC/EME-TME/64663/2006 and the PhD grant SFRH/BD/29306/2006. Acknowledgements also to the University of Minho for the provision of research facilities.

\section{References}

Abramov, O.V., 1998. High-intensity Ultrasonics: Theory and Industrial Applications, first edition. Gordon and Breach Science Publishers, Amsterdam.

Brabec, C., Mornstein, V., 2007. Detection of ultrasonic cavitation based on low frequency analysis of acoustic signal. Central European Journal of Biology 2, 213-221.

Eskin, G.I., 1998. Ultrasonic Treatment of Light Alloy Melts, first edition. Gordon and Breach Science Publishers, Amsterdam.

Fergus, J.W., 2005. Sensors for monitoring the quality of molten aluminum during casting. Journal of Materials Engineering and Performance 14 (2), 267275.

Fray, D.J., 1996. The use of solid electrolytes as sensors for applications in molten metals. Solid State Ionics 86-88, 1045-1054.

Gruzleski, J.E., Closset, B.M., 1990. The Treatment of Liquid Aluminium-Silicon Alloys, first edition. AFS, Des Plaines.

Kai, A.K., Yueh, F.Y., Singh, J.P., Zhang, H., 2002. High temperature fiber optic laser-induced breakdown spectroscopy sensor for analysis of molten alloy constituents. Review of Scientific Instruments 73, 3589-3599.

Kong, C.Y., et al., 2004. Optimum process parameters for ultrasonic consolidation of 3003 aluminium. Journal of Materials Processing Technology 146, 181187.

Makarov, S., Apelian, D., Ludwig, D., 1999. Inclusion removal and detection in molten aluminum: mechanical, electromagnetic and acoustic techniques. AFS Transactions $107,725-735$.

Matsuoka, S., Imai, H., 2009. Direct welding of different metals using ultrasonic vibration. Journal of Materials Processing Technology 209, 954-960.

Meidani, A.R.N., Hasan, M., 2004. A study of hydrogen bubble growth during ultrasonic degassing of Al-Cu alloy melts. Journal of Materials Processing Technology $147,311-320$

Ono, Y., Moisan, J.-F., Zhang, Y., Jen, C.-K., França, D.R., 2002. Development of ultrasonic techniques with buffer rod in molten aluminum. In: Proceedings of IEEE Ultrasonic Symposium , Munich, Germany, pp. 805-810.

Ono, Y., Moisan, J.-F., Zhang, Y., Jen, C.-K., Su, C.-Y., 2004. An on-line ultrasonic cleanliness analyzer for molten light metals. JOM 56 (2), 59-64.

Prokic, M., 2001. European Patent Application EP 1238715A1.

Prokic, M., 2004. Piezoelectric Converters Modeling and Characterization, second edition. MP Interconsulting, Le Locle, Switzerland.

Puga, H., et al., 2009a. Recycling of aluminium swarf by direct incorporation in aluminium melts. Journal of Materials Processing Technology 209, 51955203.

Puga, H., et al., 2009b. The influence of processing parameters on the ultrasonic degassing of molten AlSi9Cu3 aluminium alloy. Materials Letters 63, 806-808.

Puga, H., et al., 2009. The combined effect of melt stirring and ultrasonic agitation on the degassing efficiency of AlSi9Cu3 alloy. Materials Letters 63, 20892092.

Roderick, I., Guthrie, L., Li, M., 2001. In situ detection of inclusions in liquid metals. Part II. Metallurgical applications of LiMCA systems. Metallurgical and Materials Transactions B 32B, 1081-1093.

Schwandt, C., Hills, M.P., Henson, M.A., Fray, D.J., Henson, R.M., Moores, A., 2003. Determination of hydrogen in molten aluminium and its alloys using an electrochemical sensor. In: Schlesinger, M.E., TMS (Eds.), Proceedings of EDP Congress 2003. San Diego, CA, pp. 427-438.

Singh, R., Khamba, J.S., 2006. Ultrasonic machining of titanium and its alloys: a review. Journal of Materials Processing Technology 173, 125-135.

Vangrunderbeek, J., Lens, P., Castelijns, C., Verstreken, P., 1999. Continuous in-line monitoring of magnesium in aluminum. In: Light Metals, TMS, Warrendale, 1999, pp. 1005-1009.

$\mathrm{Xu}, \mathrm{H}$., et al., 2008. Effects of ultrasonic vibration on degassing of aluminum alloys. Materials Science and Engineering A 473, 96-104.

Zheng, M., Zhen, X., 1993. $\mathrm{SrCeO}_{3}$-based solid electrolyte probe sensing hydrogen content in molten aluminum. Solid State Ionics 59 (1-2), 167-169. 